\title{
A study of the Moon shadow by using GRAPES-3 muon telescope
}

M. Zuberi, ${ }^{a, *}$ S. Ahmad, ${ }^{c}$ M. Chakraborty, ${ }^{a}$ A. Chandra, ${ }^{c}$ S.R. Dugad, ${ }^{a}$ U.D. Goswami, ${ }^{l}$ S.K. Gupta, ${ }^{a}$ B. Hariharan, ${ }^{a}$ Y. Hayashi, ${ }^{b}$ P. Jagadeesan, ${ }^{a}$ A. Jain, ${ }^{a}$ P. Jain, ${ }^{d}$ S. Kawakami, ${ }^{b}$ H. Kojima, ${ }^{e}$ S. Mahapatra, ${ }^{i}$ P.K. Mohanty, ${ }^{a}$ R. Moharana, ${ }^{j}$ Y. Muraki, ${ }^{g}$ P.K. Nayak, ${ }^{a}$ T. Nonaka, ${ }^{h}$ A. Oshima,${ }^{e}$ B.P. Pant,${ }^{j}$ D. Pattanaik, ${ }^{a, i}$ G. Pradhan, ${ }^{k}$ P.S. Rakshe, ${ }^{a}$ M. Rameez, ${ }^{a}$ K. Ramesh, ${ }^{a}$ L.V. Reddy, ${ }^{a}$ R. Sahoo, ${ }^{k}$ R. Scaria, ${ }^{k}$ S. Shibata, ${ }^{e}$ K. Tanaka ${ }^{f}$ and F. Varsi ${ }^{d}$ [The GRAPES-3 Collaboration]

${ }^{a}$ Tata Institute of Fundamental Research, Homi Bhabha Road, Mumbai 400005, India

${ }^{b}$ Graduate School of Science, Osaka City University, Osaka 558-8585, Japan

${ }^{c}$ Aligarh Muslim University, Aligarh 202002, India

${ }^{d}$ Indian Institute of Technology Kanpur, Kanpur 208016, India

${ }^{e}$ College of Engineering, Chubu University, Kasugai, Aichi 487-8501, Japan

${ }^{f}$ Graduate School of Information Sciences, Hiroshima City University, Hiroshima 731-3194, Japan

${ }^{g}$ Institute for Space-Earth Environmental Research, Nagoya University, Nagoya 464-8601, Japan

${ }^{h}$ Institute for Cosmic Ray Research, Tokyo University, Kashiwa, Chiba 277-8582, Japan

${ }^{i}$ Utkal University, Bhubaneshwar 751004, India

${ }^{j}$ Indian Institute of Technology Jodhpur, Jodhpur 342037, India

${ }^{k}$ Indian Institute of Technology Indore, Indore 453552, India

${ }^{l}$ Dibrugarh University, Dibrugarh 786004, India

E-mail: meeran.zuberihep@gmail.com, pkm@tifr.res.in, and mrameezphysics@gmail.com

The GRAPES-3 experiment is designed to perform precision studies of gamma-ray sources in the TeV-PeV energy region. It consists of 400 plastic scintillator detectors spanning an effective area of $25000 \mathrm{~m}^{2}$ and a large area $\left(560 \mathrm{~m}^{2}\right)$ muon telescope which records $\sim 4 \mathrm{x} 10^{9}$ muons every day. With the recent installation of an improved triggerless data acquisition (DAQ) system, the information related to every muon is recorded with a timing resolution of $10 \mathrm{~ns}$. The angular resolution and pointing accuracy of the upgraded muon telescope has been validated by characterizing the shadow of the moon among recorded muons. Here, the details of the analysis and results, as well as the simulation studies to account for the deflection of the particles in the Earth's magnetic field will be presented.

$37^{\text {th }}$ International Cosmic Ray Conference (ICRC 2021)

July 12 th - 23rd, 2021

Online - Berlin, Germany

${ }^{*}$ Presenter 


\section{Introduction}

The atmospheric muons plays a vital role in the validation of the detector's performance and characterization of the systematic associated to the cosmic rays detector. The muons produced in the interaction of primary cosmic rays with the atmospheric nuclei can traverse deep into the atmosphere and reach the detector levels. In 1957, Clark had firstly described the shadowing effect in the cosmic ray flux by the Moon and the Sun, which can effectively be used to determine the angular resolution and the pointing accuracy of the detector [1]. However, the shadows of the Moon and the Sun can be displaced relative to the true position of these objects due to the deflection of the charge particles in the geomagnetic field (GMF) and the interplanetary magnetic field. The deflection is more for lower energy particles and with increase in energy the particles undergoes lesser deflection results in better estimation of the detector's parameters. The moon shadow has been already measured and reported by several experiments like MACRO [2], SOUDAN [3], L3+Cosmics [4], IceCube [5], and ANTARES [6]. The GRAPES-3 experiment has been also reported the $\sim 5.0 \sigma$ detection of Moon shadow effect by using extensive air shower (EAS) data, yields the angular resolution of $0.7^{\circ}$ of GRAPES-3 EAS array for $30 \mathrm{TeV}$ cosmic rays [7].

The GRAPES-3 muon telescope (G3MT) records large number of low energy muons ( $>=1 \mathrm{GeV}$ ), which suffer severe deflection by GMF as a result, it can cause the difficulty in pointing towards the real position of the Moon. Furthermore, it is known that the reconstruction accuracy of the muon tracks also depends on the intrinsic resolution of the detector. The design of the G3MT provides an average angular resolution of $4^{\circ}$, which adds up to the further challenges in determining the Moon shadow by using muons. However, we are in the process of doing major upgrade in our existing data acquisition system (DAQ) of muon angle recording by a trigger-less muon DAQ (TM-DAQ) system, which has several advantages over the existing DAQ and will discussed in the next section. In this paper, we are presenting our very first attempt to study the moon shadow effect by using preliminary data of atmospheric muons recorded by TM-DAQ.

\section{The GRAPES-3 muon telescope}

The GRAPES-3 muon telescope (G3MT) is one of the unique instrument that provides the directional measurements of the atmospheric muons. The basic element of a muon telescope is the proportional counter (PRC), which is made up of a six meter long mild steel tube with a $10 \mathrm{x} 10 \mathrm{~cm}^{2}$ cross-section, and a wall thickness of $2.3 \mathrm{~mm}$.

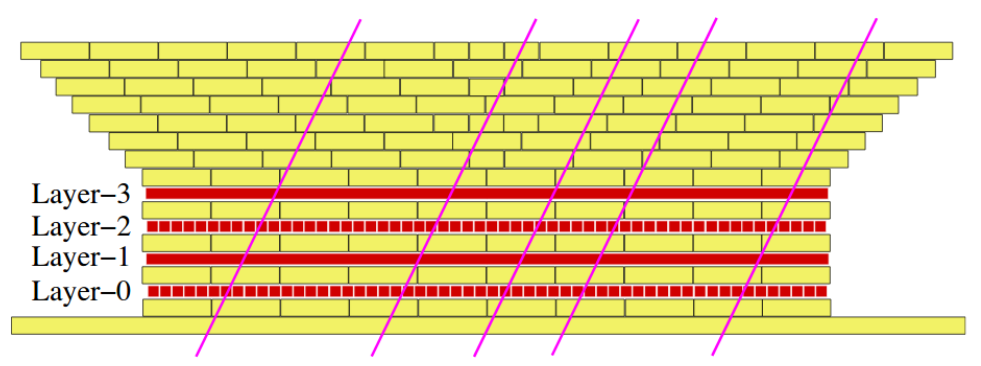

Figure 1: A schematic of the 4-layer tracking muon telescope module. 


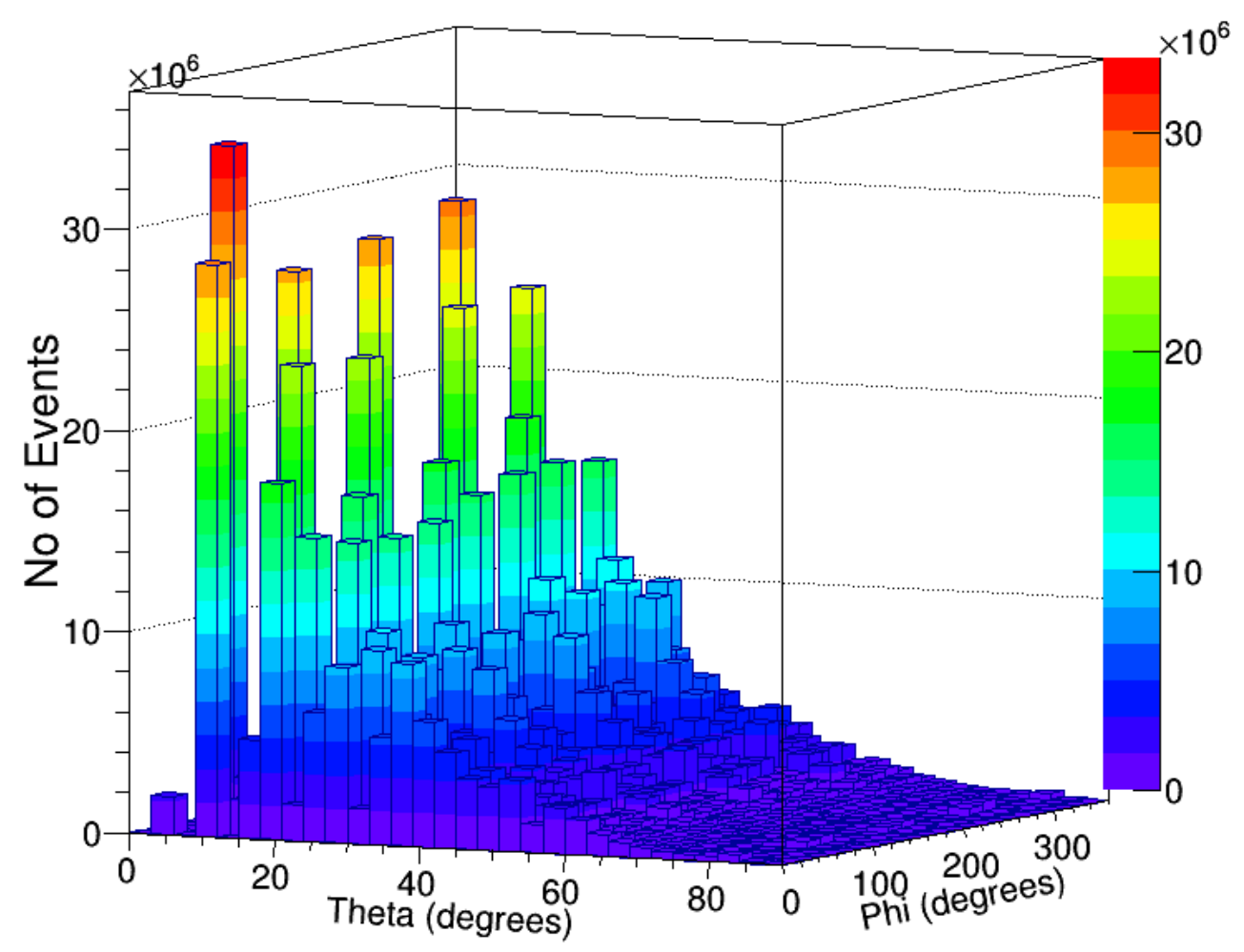

Figure 2: 2-D exposure map of recorded muons in $\theta-\phi$ space

The G3MT constitutes 16 independent muon modules having 3712 PRCs. In each module, PRCs are placed in a four-layer configuration (58 PRCs in each layer) with consecutive layers arranged in mutually perpendicular directions as shown in Fig. 1 that allow the reconstruction of each muon track in two mutually orthogonal planes with the average angular accuracy of $4^{o}$ [8]. The five pink lines drawn in Fig. 1 represents the five tracks of inclined parallel muons.

The recent upgrade in G3MT DAQ system allows to explore many interesting physics problems, which was not possible earlier due to limitations of the older DAQ. The TM-DAQ is build using FPGA based boards, which provides a large number of I/O pins that can be used for various applications. There are two highly stable crystals of $100 \mathrm{MHz}$ and $50 \mathrm{MHz}$ are installed on-board, which provide the time resolution of $10 \mathrm{~ns}$ for each PRC hit. The fast electronics of TM-DAQ also results in almost negligible dead time of $<0.0001 \%$, which was earlier $\sim 12 \%$ for the older DAQ system. More details about the TM-DAQ and its application can be found elsewhere $[9,10]$.

\section{Exposure Maps}

A 2-D exposure map in $\theta-\phi$ space is produced by using three days muon angle reconstructed data from one three modules. The Fig. 2 clearly shows the presence of large statistics up to $50^{\circ}$ beyond which also there are good amount of events recorded up to $85^{\circ}$. 


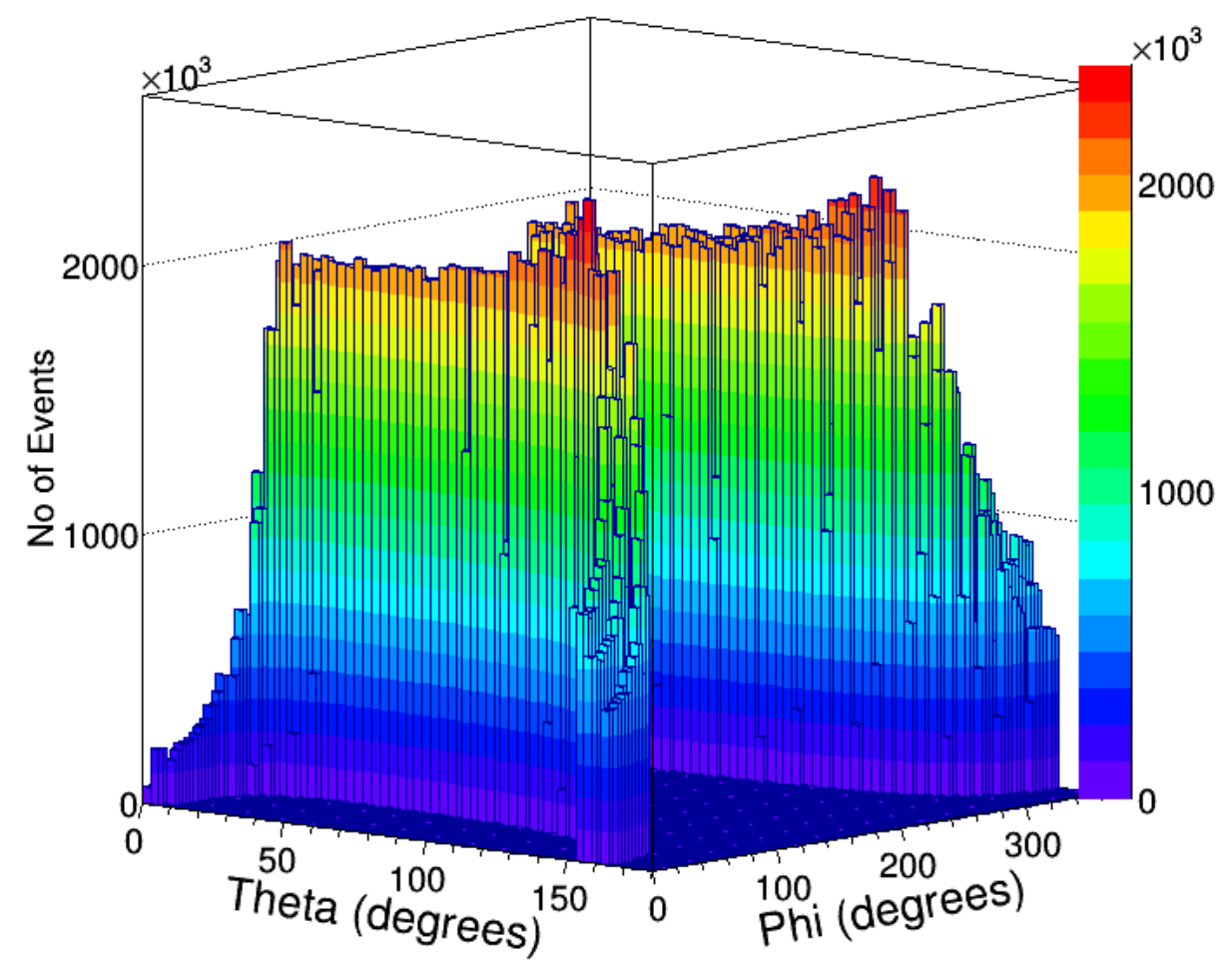

Figure 3: 2-D exposure map of the Moon in $\theta-\phi$ space

For the similar time period, the moon position is also calculated in the same $\theta-\phi$ space. Fig. 3 shows the movement of the Moon during the observational time period.

\section{Event Selection}

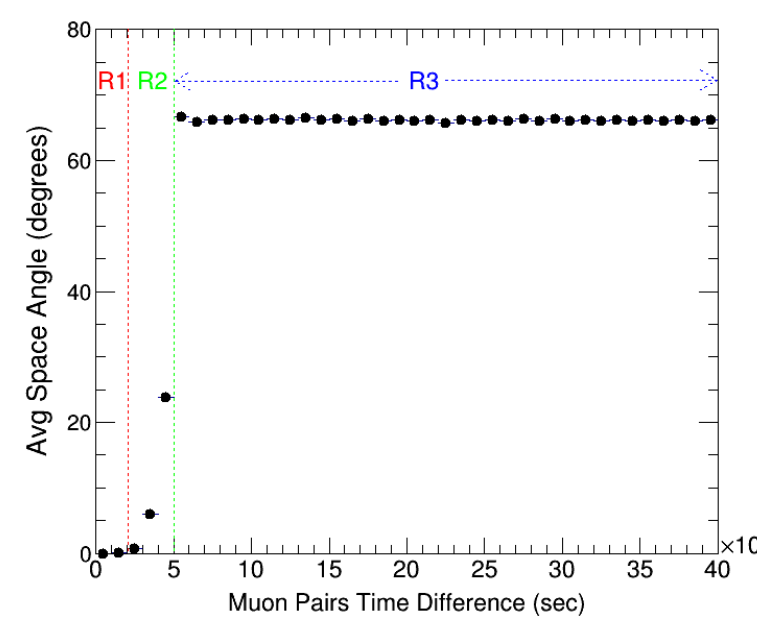

Space angle between two consecutive recorded muons as a function inter-event time difference

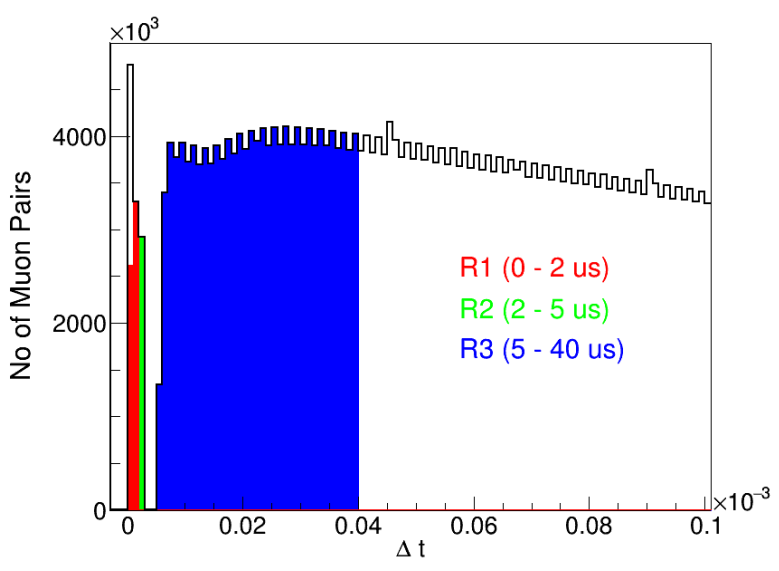

Inter-event time difference distribution 
As discussed in the previous section, the G3MT data is flooded with the low energy muons, which may undergoes to large defalcations by the GMF. Such low energy muons are filtered out by using the double muons data, which are expected to have larger energies and hence may suffer lesser deflections. The identification of such double muons is based on the hypothesis that if the two muons are recorded simultaneously and are coming from the same EAS, then the angular separation between these two muons will be minimum. The Fig. 4 shows the space angle plot between two consecutive recorded muons as a function inter-event time difference.

We have observed that the avg space angle between muon pairs remains close to zero up to $\sim 2 \mu \mathrm{sec}$ afterwards, it started to rise till $5 \mu \mathrm{sec}$ and then becomes almost constant to the value of $\sim 70^{\circ}$. Based on these observations, we have divided the data into three ranges: $\mathrm{R} 1=0-2 \mu \mathrm{sec}, \mathrm{R} 2$ $=2-5 \mu \mathrm{sec}$, and $\mathrm{R} 3=5-40 \mu \mathrm{sec}$. The number of available events in these ranges can be seen in Fig. 4, which shows that the R3 range contains the largest number of events but it is also expected to be most contaminated by low energy muons.

\section{One Dimensional Analysis}

The muon densities are plotted as a function of space angle for all three chosen ranges of R1, $\mathrm{R} 2$, and R3.

\section{Conclusions and Future Prospects}

\section{Acknowledgements}

We thank D.B. Arjunan, K. Manjunath, S. Murugapandian, S. Pandurangan, B. Rajesh, M.S. Shareef, C. Shobana, R. Sureshkumar, and other colleagues for their help in running and maintenance of the GRAPES-3 experiment.

\section{References}

[1] G.W. Clark, Phys. Rev., 108, 450 (1957).

[2] M. Ambrosio et al., Phys. Rev.D59,012003 (1999).

[3] J. H. Cobb et al., Phys. Rev.D61, 092002(2000).

[4] P. Achard et al., Astropart. Phys.23(4), 411-434 (2005).

[5] M. G. Aartsen et al., Phys. Rev.D89(10),102004 (2014)

[6] A. Albert et al., Eur. Phys. J. C (78), 1006 (2018)

[7] A. Oshima et al., Astroparticle Physics, 33, 97 (2010)

[8] Y. Hayashi et al., Nucl. Instrum. Methods A 545, 643 (2005).

[9] A. Jain et al., Proceedings of Science PoS(ICRC2021)257.

[10] B. HariHaran et al., Proceedings of Science PoS(ICRC2021)379. 